\title{
Ten-year longitudinal health-related quality of life following iodine-125 brachytherapy monotherapy for localized prostate cancer
}

\author{
Finbar Slevin, FRCRl', Brinda Sethugavalar, FRCRl,a, Bashar Al-Qaisieh, PhD², Peter Bownes, MSc², Joshua Mason, PhD2,b, \\ Jonathan Smith, FRCR ${ }^{3}$, David Bottomley, FRCR', Ann M Henry, FRCR' \\ 'Department of Clinical Oncology, Leeds Teaching Hospital NHS Trust, Leeds, UK, ${ }^{2}$ Department of Medical Physics, Leeds Teaching Hospital \\ NHS Trust, Leeds, UK, ${ }^{3}$ Department of Clinical Radiology, Leeds Teaching Hospital NHS Trust, Leeds, UK \\ Current address: 'James Cook University Hospital, Middlesbrough, UK, ' Imperial College Healthcare NHS Trust, The Bays, St Mary's Hospital, \\ London, UK
}

\begin{abstract}
Purpose: This prospective longitudinal study quantifies health-related quality of life (HRQoL) up to 10 years following permanent iodine-125 $\left({ }^{125} \mathrm{I}\right)$ prostate brachytherapy alone for localized prostate cancer.

Material and methods: In total, 120 patients completed a validated expanded prostate cancer index composite (EPIC) questionnaire pre-treatment and at 8 time points after treatment (6 weeks, 6, 10, 18 months, and 2, 3, 5, 10 years). At each time point, clinically relevant small, moderate, and severe declines in HRQoL were defined as 0.2-0.5 SD, 0.5-0.8 SD, and > 0.8 SD of baseline function for each of urinary, bowel, and sexual domains, respectively.

Results: Response rates in the first two years were $>90 \%$, but thereafter dropped to $75 \%$ and $48 \%$ at 5 and 10 years, respectively. 50 patients $(41.6 \%)$ responded at all stages. Maximal deterioration in mean urinary and sexual summary scores was noted 6 weeks after implant, with severe urinary symptoms and moderate bowel/sexual symptoms. At 6 months, urinary and bowel quality of life (QoL) had improved to mild impairment, which then fully resolved at 10 months. Sexual QoL remained mildly impaired throughout the 10 years of follow-up. At 10 years, new mild impairment of urinary and bowel QoL was found.

Conclusions: Clinically mild changes in urinary, bowel, and sexual QoL are found 10 years after ${ }^{125}$ I monotherapy. The impairment in sexual function persists from treatment, but urinary and bowel symptoms are new at 10 years.

J Contemp Brachytherapy 2020; 12, 6: 540-546 DOI: https://doi.org/10.5114/jcb.2020.101686
\end{abstract}

Key words: brachytherapy, iodine-125, quality of life, prostate cancer.

\section{Purpose}

Prostate cancer is the commonest new cancer diagnosis in men in the Western World, with approximately 48,500 new cases each year in the UK [1]. In those with early localized disease, the treatment options include active surveillance, brachytherapy, radical prostatectomy, or external beam radiotherapy [2]. The majority of men are cured of their cancer, and may have to live many years with the sequelae of treatment. It is therefore essential to have robust information about the long-term effects on urinary, bowel, hormonal, and sexual function. Few studies report long-term prospectively collected health-related quality of life (HRQoL) longitudinal data $[3,4,5,6]$.

\section{Material and methods}

\section{Patient group}

The local research ethics committee approved this study and patients gave informed written consent. From March to September 2002, 150 consecutive patients with localized prostate cancer due to undergo iodine- $125\left({ }^{125} \mathrm{I}\right)$ prostate brachytherapy were invited to complete detailed HRQoL paper questionnaires documenting urinary, bowel, sexual, and hormonal function. 126 patients participated and were requested to complete questionnaires before brachytherapy, four to 6 weeks after the implant, every 4 months in the first year, and then every 6 months in the second year as well as at the end of $3^{\text {th }}, 5^{\text {th }}$, and $10^{\text {th }}$ year. At the first two time points, patients completed the ques-
Address for correspondence: Finbar Slevin, FRCR, Leeds Teaching Hospitals NHS Trust, Bexley Wing, Beckett Street, Leeds, LS9 7TF, UK, phone: +44-113-206-7630, fax: +44-113-206-7582, 凶e-mail: finbarslevin@nhs.net
Received: 26.03.2020

Accepted: 21.09.2020

Published: 16.12.2020 
tionnaires during their routine visits to the clinic. Subsequently, patients were mailed the questionnaire, which they completed at home and returned to the research coordinator. Those patients who did not respond within two weeks of mailing were reminded by telephone. The authors have previously reported clinicopathological data and 10-year outcomes for patients treated with ${ }^{125} \mathrm{I}$ prostate brachytherapy in our center [7].

\section{HRQoL instrument}

The expanded prostate cancer index composite (EPIC) was chosen because it is a well-validated comprehensive HRQoL instrument used in previous studies. It is one of the patient-reported outcome measures (PROMs) for assessment of quality of life (QoL) included in the prostate cancer guidelines of the European Association of Urology $[2,3,8,9,10]$. The detailed questionnaires record patient-derived information on urinary, bowel, sexual, and hormonal functions within separate sub-sections or domains. Higher overall and domain scores (range, 0-100) represent better functioning and QoL, with the majority of questions using ordinal response scales (e.g., no, very small, small, moderate, big problem). It has advantages in that both a function and bother can be scored for each domain. Shorter 16- and 26-item versions of EPIC exist and are useful for clinical practice, but we wanted to obtain a greater detail of information, and therefore chose the original 50-item questionnaire.

\section{Treatment technique}

Patients who presented with a prostate volume $>50 \mathrm{ml}$ underwent three months of neo-adjuvant hormonal treatment, using either LHRH agonists or anti-androgens alone, before undergoing brachytherapy. Hormone therapy was discontinued one month after implantation. Some patients were also commenced on hormone treatment by the referring urologist before brachytherapy assessment and in this situation, hormone manipulation was also discontinued one month after brachytherapy.

All patients were treated by implantation with ${ }^{125} \mathrm{I}$ seeds as monotherapy. Planning objectives were a minimum peripheral dose of 145 Gy (prescription dose according to TG43) to the prostate capsule plus a margin of 2 to $5 \mathrm{~mm}$, a $\mathrm{Vp}_{100}$ (prostate volume covered by $100 \%$ of the prescription dose) of greater than $99 \%$, and a $D_{90}$ (dose to $90 \%$ of the prostate) of approximately $180 \mathrm{~Gy}$ $(124 \%)$. The $\mathrm{D}_{90}$ planned was expected to be higher than that achieved. Rectal dose constraints were mucosa $V_{100}$ $<0.9 \mathrm{cc}$, but no formal urethral dose constraints were used. Patients were treated with either a two-step or single-step pre-planning technique using Seattle method, and prostate volume was estimated using TRUS to plan the treatment [11]. All implants were carried out using RAPID Strand ${ }^{\mathrm{TM}}$ (ONCURA, Arlington Heights, USA) stranded seeds. Post-implant dosimetry was performed by computed tomography (CT) at 6 to 8 weeks after implantation, when the majority of edema should have settled. Patients were not catheterized for post-implant CT dosimetry.

\section{Statistical analysis}

Overall and individual symptom-domain scores were recorded for each patient. Descriptive statistics (mean, standard deviation, and confidence intervals) were used to document HRQoL at each time point. Mean values at each time point were compared with the baseline values using $95 \%$ confidence intervals (95\% CI), and statistical significance was compared using a paired $t$-test. No imputation of missing data was performed. A $p$ value of $<0.05$ was taken to indicate a statistically significant difference. However, to identify clinically relevant changes in HRQoL, small, moderate, and severe declines were defined as 0.2-0.5 SD, 0.5-0.8 SD, and > 0.8 SD of baseline function for each of urinary, bowel, and sexual domains, respectively $[12,13,14]$. Regression analysis was applied to quantify any relationship between dosimetry parameters and EPIC QoL scores.

\section{Results}

At pre-treatment, 126 out of the 150 invited participants completed the questionnaires. On review, 6 of these had undergone external beam radiotherapy with ${ }^{125} \mathrm{I}$ boost, and were therefore excluded from this analysis. All of the remaining 120 patients presented with lowor intermediate-risk localized prostate cancer based on ESTRO/EAU/EORTC consensus guideline recommendations, apart from one patient who had small volume Gleason 8, as per the International Society of Urologic Pathology (ISUP) grade 4 disease [15]. Mean age at the treatment was 63.9 (range, 43-76) years. Over half of all patients received neo-adjuvant hormone manipulation $(62 \%, 74$ patients); the majority for gland reduction but in some cases, it started prior to the referral for brachytherapy by the referring urologist.

Table 1 presents all responses per domain over the 10 -year study period with HRQoL expressed in mean (SD). Table 2 shows the proportion of patients with small (0.2-0.5 SD), moderate (0.5-0.8 SD), and large (> 0.8 SD) declines in HRQoL for urinary, bowel, sexual, and hormonal domains at each time point up to 5 years. Questionnaire response rates in the first two years were $>90 \%$, but thereafter dropped to $75 \%$ and $48 \%$ at 5 and 10 years, respectively. 50 patients $(41.6 \%)$ responded at all stages. Response rates of the initial responders were at least $75 \%$ at each of the time points, with 77 patients responding at all time points.

\section{Urinary function}

As reported in our previously published work [16], a maximum deterioration was seen in urinary summary scores 6 weeks after implant. The drop in QoL was consistent across all four domains of function, bother, incontinence, and irritative or obstructive symptoms. At 6 months, urinary QoL had improved but remained statistically significantly lower than baseline (Table 1 and Figure 1). By 10 months, the overall analysis showed urinary summary scores had returned to pre-treatment levels. Up to 18 months post-implant, reduced urinary QoL compared to baseline was seen, but at 2 years, symptoms 
Table 1. The number of patients responding to expanded prostate cancer index composite (EPIC) health-related quality of life (HRQoL) questionnaire for each time point over 10 years, with all responses per domain in the study period expressed as group means (SD)

\begin{tabular}{|c|c|c|c|c|c|c|c|c|c|c|}
\hline \multirow[t]{2}{*}{ Variables } & \multirow{2}{*}{$\begin{array}{l}\text { Pre- } \\
\text { treatment }\end{array}$} & \multirow{2}{*}{$\begin{array}{l}0.2 \text { SD } \\
0.5 \text { SD } \\
0.8 \text { SD }\end{array}$} & \multicolumn{8}{|c|}{ Time point } \\
\hline & & & $\begin{array}{c}6 \\
\text { weeks }\end{array}$ & $\begin{array}{c}6 \\
\text { months }\end{array}$ & $\begin{array}{c}10 \\
\text { months }\end{array}$ & $\begin{array}{c}18 \\
\text { months }\end{array}$ & $\begin{array}{c}2 \\
\text { years }\end{array}$ & $\begin{array}{c}3 \\
\text { years }\end{array}$ & $\begin{array}{c}5 \\
\text { years }\end{array}$ & $\begin{array}{c}10 \\
\text { years }\end{array}$ \\
\hline No. of patients & 120 & & 110 & 116 & 115 & 113 & 111 & 96 & 90 & 57 \\
\hline$\%$ of baseline & 100 & & 92 & 97 & 96 & 94 & 93 & 80 & 75 & 48 \\
\hline \multicolumn{11}{|c|}{ Mean urinary (SD) } \\
\hline Summary & $\begin{array}{c}88.1 \\
(11.8)\end{array}$ & $\begin{array}{c}2.36 \\
5.9 \\
9.44 \\
\end{array}$ & $\begin{array}{l}65.3^{\star \star} \\
(25.4)\end{array}$ & $\begin{array}{l}82.8^{*} \\
(2.6)\end{array}$ & $\begin{array}{c}87.5 \\
(10.4)\end{array}$ & $\begin{array}{c}87.0 \\
(10.3)\end{array}$ & $\begin{array}{l}86.9 \\
(7.3)\end{array}$ & $\begin{array}{l}89.0 \\
(11.2)\end{array}$ & $\begin{array}{c}89.8 \\
(10.4)\end{array}$ & $\begin{array}{c}85^{*} \\
(16.0)\end{array}$ \\
\hline Function & $\begin{array}{l}94.6 \\
(9.5)\end{array}$ & $\begin{array}{c}1.9 \\
4.75 \\
7.6 \\
\end{array}$ & $\begin{array}{c}73.7^{\star \star} \\
(27.3)\end{array}$ & $\begin{array}{l}90.3^{*} \\
(12.1)\end{array}$ & $\begin{array}{l}93.3 \\
(8.7)\end{array}$ & $\begin{array}{l}90.4^{*} \\
(12.0)\end{array}$ & $\begin{array}{l}93.5 \\
(6.4)\end{array}$ & $\begin{array}{c}93.7 \\
(10.2)\end{array}$ & $\begin{array}{l}94.8 \\
(9.6)\end{array}$ & $\begin{array}{c}92^{*} \\
(13.0)\end{array}$ \\
\hline Bother & $\begin{array}{c}83.3 \\
(15.1)\end{array}$ & $\begin{array}{c}3.0 \\
7.55 \\
12.08 \\
\end{array}$ & $\begin{array}{l}58.1^{* *} \\
(25.1)\end{array}$ & $\begin{array}{l}77.0^{*} \\
(15.6)\end{array}$ & $\begin{array}{l}83.2 \\
(13.4)\end{array}$ & $\begin{array}{l}83.6 \\
(12.4)\end{array}$ & $\begin{array}{l}81.8 \\
(9.6)\end{array}$ & $\begin{array}{c}85.2 \\
(13.9)\end{array}$ & $\begin{array}{l}85.6 \\
(13.9)\end{array}$ & $\begin{array}{c}82 \\
(16.0)\end{array}$ \\
\hline Incontinence & $\begin{array}{r}92.9 \\
(11.8)\end{array}$ & $\begin{array}{c}2.36 \\
5.9 \\
9.44\end{array}$ & $\begin{array}{l}78.5^{\star \star} \\
(35.5)\end{array}$ & $\begin{array}{l}90.4^{*} \\
(13.7)\end{array}$ & $\begin{array}{c}93.5 \\
(13.2)\end{array}$ & $\begin{array}{l}92.4 \\
(9.8)\end{array}$ & $\begin{array}{l}94.6 \\
(7.0)\end{array}$ & $\begin{array}{c}91.2 \\
(16.5)\end{array}$ & $\begin{array}{c}92.0 \\
(14.7)\end{array}$ & $\begin{array}{c}87^{\star} \\
(22.0)\end{array}$ \\
\hline $\begin{array}{l}\text { Irritative/ } \\
\text { obstructive }\end{array}$ & $\begin{array}{l}86.6 \\
(12.8)\end{array}$ & $\begin{array}{c}2.56 \\
6.4 \\
10.24 \\
\end{array}$ & $\begin{array}{l}60.0^{\star *} \\
(25.7)\end{array}$ & $\begin{array}{c}80.0^{\star *} \\
(14.9)\end{array}$ & $\begin{array}{l}84.9 \\
(12.3)\end{array}$ & $\begin{array}{c}84.8 \\
(12.6)\end{array}$ & $\begin{array}{l}83.8^{*} \\
(9.3)\end{array}$ & $\begin{array}{c}88.6 \\
(10.6)\end{array}$ & $\begin{array}{c}89.6 \\
(10.3)\end{array}$ & $\begin{array}{c}85 \\
(17.0)\end{array}$ \\
\hline \multicolumn{11}{|c|}{ Mean bowel (SD) } \\
\hline Summary & $\begin{array}{c}92.6 \\
(12.3)\end{array}$ & $\begin{array}{l}2.46 \\
6.15 \\
9.84 \\
\end{array}$ & $\begin{array}{c}84.7^{\star \star} \\
(14.9)\end{array}$ & $\begin{array}{l}88.9^{*} \\
(11.8)\end{array}$ & $\begin{array}{l}91.7 \\
(8.4)\end{array}$ & $\begin{array}{c}91.3 \\
(10.3)\end{array}$ & $\begin{array}{l}93.0 \\
(5.7)\end{array}$ & $\begin{array}{l}92.4 \\
(9.5)\end{array}$ & $\begin{array}{l}94.1 \\
(7.4)\end{array}$ & $\begin{array}{c}90^{*} \\
(13.0)\end{array}$ \\
\hline Function & $\begin{array}{l}93.2 \\
(9.2)\end{array}$ & $\begin{array}{c}1.84 \\
4.6 \\
7.36 \\
\end{array}$ & $\begin{array}{l}85.3^{\star *} \\
(14.2)\end{array}$ & $\begin{array}{l}89.8^{\star} \\
(11.0)\end{array}$ & $\begin{array}{l}92.0 \\
(7.8)\end{array}$ & $\begin{array}{l}92.2 \\
(6.7)\end{array}$ & $\begin{array}{l}93.2 \\
(5.3)\end{array}$ & $\begin{array}{l}92.8 \\
(8.9)\end{array}$ & $\begin{array}{l}94.5 \\
(6.8)\end{array}$ & $\begin{array}{c}93 \\
(10.0)\end{array}$ \\
\hline Bother & $\begin{array}{c}93.5 \\
(10.5)\end{array}$ & $\begin{array}{c}2.1 \\
5.25 \\
8.4 \\
\end{array}$ & $\begin{array}{l}84.1^{\star \star} \\
(16.9)\end{array}$ & $\begin{array}{c}87.6^{\star \star} \\
(14.5)\end{array}$ & $\begin{array}{c}91.4 \\
(10.4)\end{array}$ & $\begin{array}{l}91.4 \\
(8.8)\end{array}$ & $\begin{array}{l}90.2^{*} \\
(11.2)\end{array}$ & $\begin{array}{c}91.9 \\
(11.3)\end{array}$ & $\begin{array}{l}93.8 \\
(9.2)\end{array}$ & $\begin{array}{c}90^{*} \\
(16.0)\end{array}$ \\
\hline \multicolumn{11}{|c|}{ Mean sexual (SD) } \\
\hline Summary & $\begin{array}{c}44.6 \\
(29.4)\end{array}$ & $\begin{array}{c}5.88 \\
14.7 \\
23.52 \\
\end{array}$ & $\begin{array}{l}27.0^{\star *} \\
(25.0)\end{array}$ & $\begin{array}{l}33.3^{*} \\
(24.6)\end{array}$ & $\begin{array}{l}37.4^{\star} \\
(23.4)\end{array}$ & $\begin{array}{l}32.7^{\star} \\
(21.3)\end{array}$ & $\begin{array}{l}36.3^{*} \\
(17.7)\end{array}$ & $\begin{array}{c}40.3 \\
(25.8)\end{array}$ & $\begin{array}{l}36.0^{*} \\
(25.1)\end{array}$ & $\begin{array}{c}33^{*} \\
(24.0)\end{array}$ \\
\hline Function & $\begin{array}{c}37.9 \\
(31.5)\end{array}$ & $\begin{array}{c}6.3 \\
15.75 \\
25.2 \\
\end{array}$ & $\begin{array}{l}20.6^{\star \star} \\
(25.4)\end{array}$ & $\begin{array}{l}26.9^{*} \\
(25.1)\end{array}$ & $\begin{array}{l}31.3^{*} \\
(23.7)\end{array}$ & $\begin{array}{l}26.9^{*} \\
(21.7)\end{array}$ & $\begin{array}{l}30.7^{\star} \\
(17.2)\end{array}$ & $\begin{array}{c}34.9 \\
(26.2)\end{array}$ & $\begin{array}{l}28.2^{*} \\
(25.4)\end{array}$ & $\begin{array}{c}24^{*} \\
(25.0)\end{array}$ \\
\hline Bother & $\begin{array}{c}60.7 \\
(37.5)\end{array}$ & $\begin{array}{c}7.5 \\
18.75 \\
30 \\
\end{array}$ & $\begin{array}{l}42.0^{*} \\
(39.4)\end{array}$ & $\begin{array}{l}48.3^{*} \\
(37.0)\end{array}$ & $\begin{array}{l}51.5^{\star} \\
(33.1)\end{array}$ & $\begin{array}{l}45.1^{*} \\
(29.7)\end{array}$ & $\begin{array}{l}49.2^{*} \\
(22.6)\end{array}$ & $\begin{array}{l}52.8^{*} \\
(33.3)\end{array}$ & $\begin{array}{l}52.5^{\star} \\
(36.0)\end{array}$ & $\begin{array}{c}55 \\
(38.0)\end{array}$ \\
\hline \multicolumn{11}{|c|}{ Mean hormonal (SD) } \\
\hline Summary & $\begin{array}{c}83.9 \\
(15.9)\end{array}$ & $\begin{array}{c}3.18 \\
7.95 \\
12.72 \\
\end{array}$ & $\begin{array}{c}81.2 \\
(15.2)\end{array}$ & $\begin{array}{c}87.6 \\
(12.8)\end{array}$ & $\begin{array}{l}87.8 \\
(11.1)\end{array}$ & $\begin{array}{l}90.5 \\
(9.9)\end{array}$ & $\begin{array}{l}93.0 \\
(9.5)\end{array}$ & $\begin{array}{c}91.9 \\
(10.5)\end{array}$ & $\begin{array}{c}90.6 \\
(11.4)\end{array}$ & $\begin{array}{c}89 \\
(13.0)\end{array}$ \\
\hline Function & $\begin{array}{c}79.5 \\
(19.4)\end{array}$ & $\begin{array}{c}3.88 \\
9.7 \\
15.52\end{array}$ & $\begin{array}{c}76.6 \\
(18.8)\end{array}$ & $\begin{array}{l}85.4 \\
(14.5)\end{array}$ & $\begin{array}{c}85.7 \\
(12.8)\end{array}$ & $\begin{array}{c}89.0 \\
(10.7)\end{array}$ & $\begin{array}{l}92.4 \\
(5.7)\end{array}$ & $\begin{array}{l}90.9 \\
(11.6)\end{array}$ & $\begin{array}{c}89.4 \\
(12.7)\end{array}$ & $\begin{array}{c}90 \\
(19.0)\end{array}$ \\
\hline Bother & $\begin{array}{c}87.1 \\
(14.6)\end{array}$ & $\begin{array}{c}2.92 \\
7.3 \\
11.68\end{array}$ & $\begin{array}{c}85.2 \\
(14.1)\end{array}$ & $\begin{array}{l}90.5 \\
(11.1)\end{array}$ & $\begin{array}{c}90.0 \\
(10.8)\end{array}$ & $\begin{array}{c}91.6 \\
(10.4)\end{array}$ & $\begin{array}{l}94.9 \\
(5.6)\end{array}$ & $\begin{array}{c}92.9 \\
(10.3)\end{array}$ & $\begin{array}{c}91.6 \\
(11.3)\end{array}$ & $\begin{array}{c}90 \\
(14.0)\end{array}$ \\
\hline
\end{tabular}

${ }^{*}$ SD of group mean 0.5-0.8, ${ }^{*}$ SD of group mean $>0.8$ 
Table 2. The percentage of patients responding to expanded prostate cancer index composite (EPIC) healthrelated quality of life $(\mathrm{HRQOL})$ questionnaire for each time point over 5 years, with small (0.2-0.5 SD), moderate (0.5-0.8 SD), and severe (>0.8 SD) declines from baseline function

\begin{tabular}{|c|c|c|c|c|c|c|c|c|c|}
\hline \multirow[t]{2}{*}{ Variables } & \multirow{2}{*}{$\begin{array}{c}\text { Pre- } \\
\text { treatment } \\
\text { score }\end{array}$} & & \multicolumn{7}{|c|}{ Time point } \\
\hline & & & $\begin{array}{c}6 \\
\text { weeks }\end{array}$ & $\begin{array}{c}6 \\
\text { months }\end{array}$ & $\begin{array}{c}10 \\
\text { months }\end{array}$ & $\begin{array}{c}18 \\
\text { months }\end{array}$ & $\begin{array}{c}2 \\
\text { years }\end{array}$ & $\begin{array}{c}3 \\
\text { years } \\
\end{array}$ & $\begin{array}{c}5 \\
\text { years } \\
\end{array}$ \\
\hline No. of patients & 120 & & 110 & 116 & 115 & 113 & 111 & 96 & 90 \\
\hline$\%$ of baseline & 100 & & 92 & 97 & 96 & 94 & 93 & 80 & 75 \\
\hline Mean (SD) & & & \multicolumn{7}{|c|}{ Percentage of patients per group at each time point (\%) } \\
\hline $\begin{array}{l}\text { Urinary } \\
\text { summary }\end{array}$ & $88.1(11.8)$ & $\begin{array}{c}<0.2 S D \\
0.2-0.5 S D \\
0.5-0.8 S D \\
>0.8 S D\end{array}$ & $\begin{array}{c}19.1 \\
7.3 \\
8.2 \\
65.5\end{array}$ & $\begin{array}{c}45.7 \\
6.0 \\
10.3 \\
37.9\end{array}$ & $\begin{array}{c}53.9 \\
8.7 \\
13.9 \\
23.5\end{array}$ & $\begin{array}{c}56.6 \\
7.1 \\
10.6 \\
25.7\end{array}$ & $\begin{array}{l}47.7 \\
11.7 \\
16.2 \\
24.3\end{array}$ & $\begin{array}{c}70.8 \\
7.3 \\
7.3 \\
14.6\end{array}$ & $\begin{array}{c}67.7 \\
12.9 \\
7.5 \\
11.8\end{array}$ \\
\hline $\begin{array}{l}\text { Bowel } \\
\text { summary }\end{array}$ & $92.6(12.3)$ & $\begin{array}{c}<0.2 \mathrm{SD} \\
0.2-0.5 \mathrm{SD} \\
0.5-0.8 \mathrm{SD} \\
>0.8 \mathrm{SD}\end{array}$ & $\begin{array}{l}34.5 \\
15.5 \\
13.6 \\
36.4\end{array}$ & $\begin{array}{c}52.6 \\
11.2 \\
6.9 \\
29.3\end{array}$ & $\begin{array}{l}60.0 \\
12.2 \\
15.7 \\
12.2\end{array}$ & $\begin{array}{c}46.9 \\
21.2 \\
23.0 \\
8.8\end{array}$ & $\begin{array}{c}55.9 \\
26.1 \\
12.6 \\
5.4\end{array}$ & $\begin{array}{c}66.7 \\
12.5 \\
6.3 \\
14.6\end{array}$ & $\begin{array}{c}76.3 \\
8.6 \\
8.6 \\
6.5\end{array}$ \\
\hline $\begin{array}{l}\text { Sexual } \\
\text { summary }\end{array}$ & $44.6(29.4)$ & $\begin{array}{c}<0.2 S D \\
0.2-0.5 \mathrm{SD} \\
0.5-0.8 \mathrm{SD} \\
>0.8 \mathrm{SD}\end{array}$ & $\begin{array}{c}43.6 \\
13.6 \\
5.5 \\
37.3\end{array}$ & $\begin{array}{c}87.1 \\
12.9 \\
0.0 \\
0.0\end{array}$ & $\begin{array}{c}94.8 \\
5.2 \\
0.0 \\
0.0\end{array}$ & $\begin{array}{l}91.2 \\
8.8 \\
0.0 \\
0.0\end{array}$ & $\begin{array}{c}96.4 \\
3.6 \\
0.0 \\
0.0\end{array}$ & $\begin{array}{l}88.5 \\
11.5 \\
0.0 \\
0.0\end{array}$ & $\begin{array}{l}88.6 \\
11.4 \\
0.0 \\
0.0\end{array}$ \\
\hline $\begin{array}{l}\text { Hormonal } \\
\text { summary }\end{array}$ & $83.9(15.9)$ & $\begin{array}{c}<0.2 S D \\
0.2-0.5 \mathrm{SD} \\
0.5-0.8 \mathrm{SD} \\
>0.8 \mathrm{SD}\end{array}$ & $\begin{array}{c}61.8 \\
10.0 \\
9.1 \\
19.1\end{array}$ & $\begin{array}{c}73.3 \\
9.5 \\
9.5 \\
7.8\end{array}$ & $\begin{array}{c}70.4 \\
12.2 \\
8.7 \\
8.7\end{array}$ & $\begin{array}{c}77.9 \\
13.3 \\
2.7 \\
6.2\end{array}$ & $\begin{array}{c}83.8 \\
9.9 \\
5.4 \\
0.9\end{array}$ & $\begin{array}{c}87.5 \\
4.2 \\
5.2 \\
3.1\end{array}$ & $\begin{array}{c}75.3 \\
9.7 \\
5.4 \\
9.7\end{array}$ \\
\hline
\end{tabular}

10-year time point data not available

returned to baseline and were maintained up to 5 years. At 10 years post-treatment, a mild reduction in urinary function (0.5-0.8 SD of group mean) was noted, with a small increase in urinary incontinence reported.

\section{Sexual function}

Baseline sexual QoL scores for our patients were low, with a mean summary score of 44.6 and a sexual function

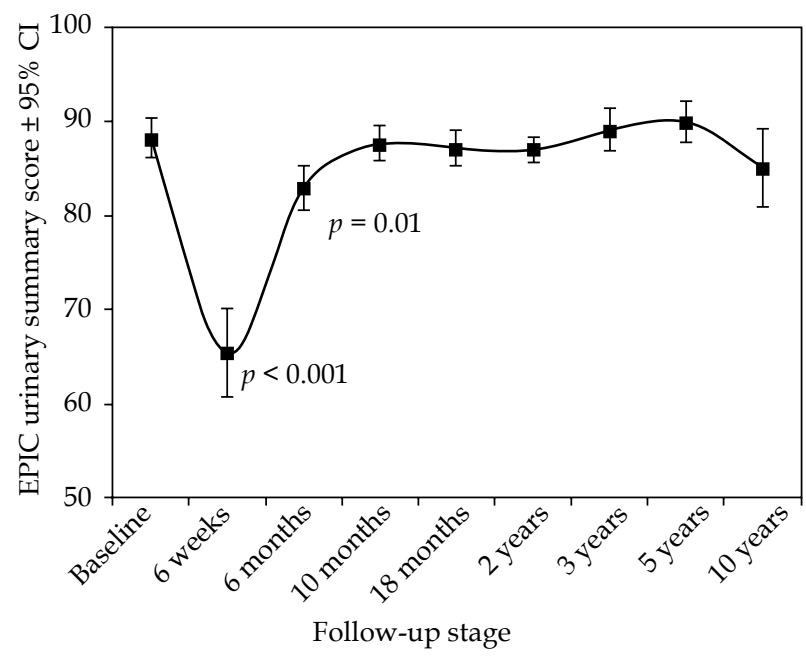

Fig. 1. The change in mean expanded prostate cancer index composite (EPIC) urinary summary score in 10 years following ${ }^{125} \mathrm{I}$ brachytherapy for all patients. $P$-value only shown if $p<0.05$, with the comparison made to quality of life (QoL) at baseline score of 37.7. The mean sexual summary and the subscales of sexual function and bother never returned to the pre-treatment levels (Table 1 and Figure 2). Interestingly, the average bother score was higher at baseline at 60.7, and remained proportionally higher than the function score suggesting that the observed reduction in sexual function was accepted by this cohort of patients (Table 1). Increasing age was an independent prognostic factor at

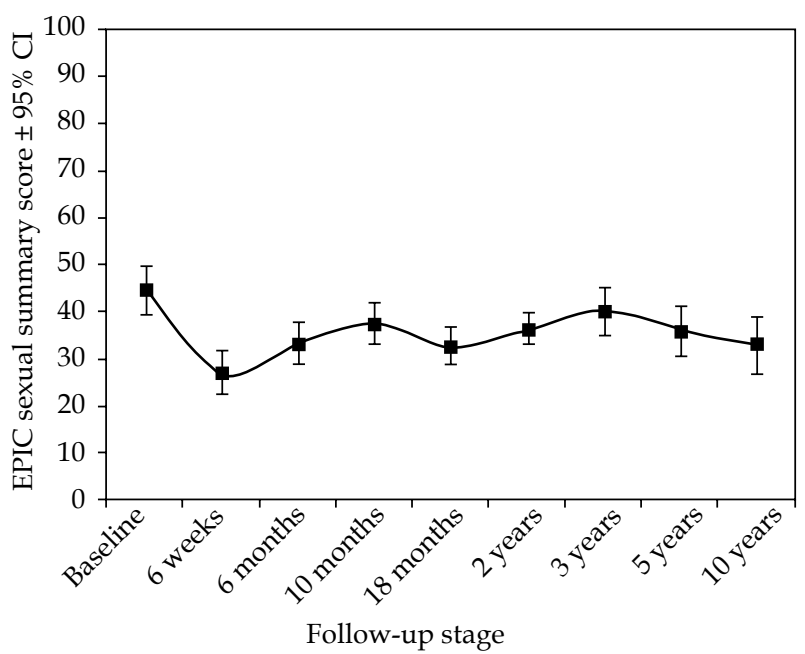

Fig. 2. The change in mean expanded prostate cancer index composite (EPIC) sexual summary score in 10 years following ${ }^{125} \mathrm{I}$ brachytherapy 


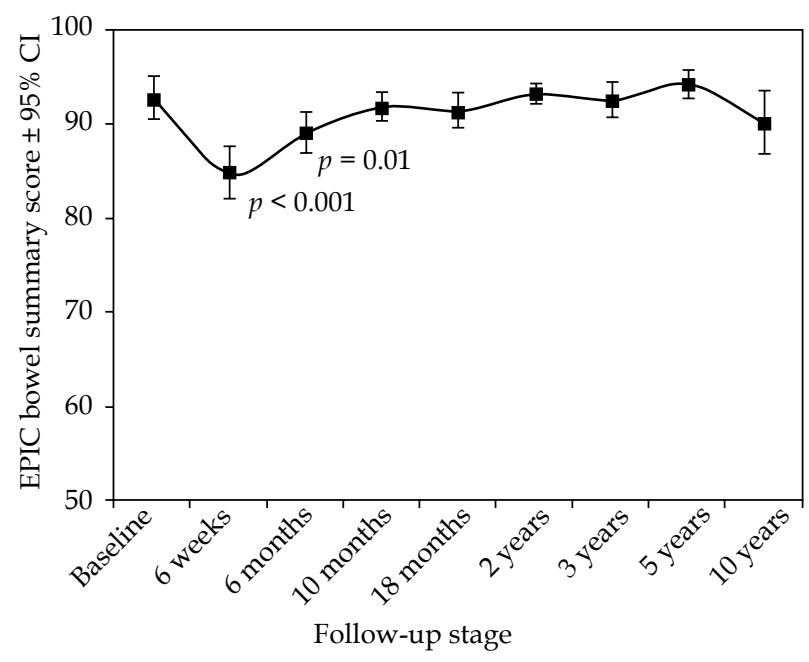

Fig. 3. The change in mean expanded prostate cancer index composite (EPIC) bowel summary score in 10 years following ${ }^{125} \mathrm{I}$ brachytherapy. $P$-value only shown if $p<0.05$, with the comparison made to quality of life (QoL) at baseline

all time points for the mean summary and the sub-scale function, but not for the sub-scale bother $(p<0.01)$.

\section{Bowel function}

A small impact on bowel QoL was noted at 6 weeks, which returned to baseline within 6 to 10 months of implantation for all patients. A small increase in bothering bowel symptoms (0.5-0.8 SD of group mean) was noted at 10 years, although bowel function remained stable (Table 1 and Figure 3).

\section{Post-implant dosimetry}

Computed tomography post-implant dosimetry was available in 110 out of 120 patients (91.7\%). CT post-implant dosimetry was undertaken at 6 weeks without catheterization. The mean (SD) $\mathrm{D}_{90}$ was 146.1 (19) Gy. Using regression analysis, there was no correlation between mean central dose (a surrogate for urethral position in un-catheterized patients), prostate $\mathrm{V}_{150}$, or $\mathrm{V}_{200}$ as well as the EPIC urinary summary score (or any of the subscores). Also, there was no relationship between the volume of rectum receiving $100 \%$ or $68 \%$ of the prescription dose $\left(\mathrm{Vr}_{100}\right.$ or $\left.\mathrm{Vr}_{68}\right)$ and the EPIC bowel scores.

\section{Discussion}

This prospective longitudinal study demonstrates that after an initial period of mild to moderate symptoms, permanent prostate brachytherapy using ${ }^{125} \mathrm{I}$ was well tolerated with a little deterioration in long-term QoL.

The most robust information about long-term QoL following treatment for prostate cancer comes from randomized trials where intervention groups have balanced baseline characteristics. A recent systematic review was published of randomized controlled trials and non-randomized comparative studies of QoL after prostatecto- my, brachytherapy, and external beam radiotherapy in patients with localized prostate cancer [17]. The authors concluded that brachytherapy patients mainly reported irritative/obstructive urinary problems up to around 1 year, after which QoL gradually returned to baseline.

Attempts to recruit patients into phase III trials comparing radical prostatectomy versus interstitial brachytherapy have been limited, as a significant proportion of informed patients feel unable to commit to a random allocation of treatment. An early terminated SPIRIT trial [3] showed a comparison of QoL at a median of 5.2 years after treatment with either prostatectomy or brachytherapy (no neo-adjuvant hormone use). This cross-sectional study assessed 168 study eligible men, 3.2 to 6.5 years after treatment, and demonstrated that those patients who underwent brachytherapy had better urinary, sexual, and patient satisfaction scores than men undergoing radical prostatectomy. The patients in the SPIRIT study had a mean 5-year EPIC sexual summary of 52.5, which is significantly higher than the mean 5-year EPIC sexual summary score of 36 for our patients. This may be because our group was an unselected cohort of consecutively treated patients. In addition, our patients were older, with mean age of 63.9 years compared to an average age of 61.4 years for patients treated with brachytherapy in the SPIRIT study. Furthermore, a number of our patients would likely not have been suitable for prostatectomy because of co-morbidity.

Giberti et al. randomized 200 patients to either prostatectomy or brachytherapy and assessed QoL at 6 months, 1 year, and 5 years after treatment, although alternative QoL questionnaires to EPIC were used [18]. While patients treated with brachytherapy reported more irritative urinary symptoms at 6 months and 1 year, a better erectile function was also reported at these time points by brachytherapy patients. By 5 years, the authors found no difference between the groups in terms of urinary disorders and sexual activity. However, this study did not assess QoL between 1 and 5 years.

Hoffman et al. prospectively evaluated PROMs over 5 years using the 26-item EPIC questionnaire in patients with favorable-risk prostate cancer managed with active surveillance, prostatectomy, external beam radiotherapy (EBRT), and brachytherapy (although patients treated with brachytherapy comprised only $6 \%$ of the cohort) [5]. For patients treated with brachytherapy, the authors observed worse urinary, sexual, and bowel function at 1 year compared with active surveillance, but by 5 years, most functional differences across all active treatments had attenuated. However, patients treated with prostatectomy reported worse urinary incontinence throughout 5 years compared with all other treatment options.

The prostate testing for cancer and treatment (ProtecT) study compared outcomes after prostatectomy, EBRT, and active monitoring for 1,643 patients diagnosed with localized prostate cancer after prostate specific antigen (PSA) testing [4]. Evaluation of PROMs demonstrated that prostatectomy had a greater initial and persisting effect on urinary continence than radiotherapy or active monitoring. Urinary incontinence after prostatectomy was also associated with reduction in QoL for up to 
2 years. Initial and persisting erectile dysfunction were also worse after prostatectomy than radiotherapy.

Prospective longitudinal studies comparing non-randomized cohorts of patients undergoing prostatectomy, brachytherapy, or external beam radiotherapy without hormone manipulation demonstrated that 3 years after treatment, brachytherapy patients experienced less urinary incontinence, bowel effects, and sexual dysfunction, but more urinary irritative-obstructive symptoms [9,19]. This could indicate a tendency in overtime reduction of sexual function in brachytherapy patients that may be related to increasing age and/or a late effect of radiation [9]. Sanda et al. included men who underwent neo-adjuvant hormone treatment into a multi-center prospective longitudinal study of all three treatment modalities [10]. The use of hormone manipulation in brachytherapy patients was associated with more sexual dysfunction and hormonal symptoms in the first 12 months after treatment, but by two years, sexual functioning had returned to a level similar to that of the cohort who had brachytherapy alone.

A single-center prospective longitudinal study using RAND-36 generic health survey, EORTC core questionnaire QLQ-C30, and tumor-specific EORTC prostate cancer module (EORTC-PR25) in a cohort of 127 patients undergoing ${ }^{125} \mathrm{I}$ brachytherapy demonstrated that after the first year, urinary and bowel symptoms returned to baseline and remained stable up to 6 years after treatment [20]. Similar to our cohort, sexual activity scores had diminished by 6 years in $70 \%$ of the patients treated in the absence of a significant reduction in sexual functioning. Indeed, emotional functioning scores had significantly improved at 6 years suggesting that although men may be less sexually active as time goes on, they may be less bothered about it.

Evers et al. [21] prospectively evaluated the effect of neo-adjuvant hormones on urinary function (using the international prostate symptoms scores) and global QoL (EORTC-QLQ) in a cohort of 40 patients having ${ }^{125} \mathrm{I}$ monotherapy with a follow-up period of 1 year. At baseline, the neo-adjuvant group reported worse urinary function, but experienced greater improvement in urinary function at 3 months when compared to those not receiving neo-adjuvant hormones. Other prospective longitudinal QoL studies suggested that short-term neoadjuvant hormones did not appear to significantly affect long-term urinary or sexual function in brachytherapy patients [22]

At 10 years, we found small declines in urinary and bowel QoL. The relationship between the development of urinary and bowel symptoms at 10 years and brachytherapy was not clear in our study, but it could be as a consequence of increasing patient age or a late effect of brachytherapy. The initial declines in urinary and bowel QoL had resolved to baseline by 2 years and 6-10 months, respectively, and urinary incontinence was noted at 10 years rather than irritative/obstructive symptoms generally associated with brachytherapy. However, late urinary toxicity is recognized, and a study by Keyes et al. of 2,011 patients found prevalence of RTOG grade $\geq 2$ to be $8.8 \%$ from 7 to 13 years post-brachytherapy. Greater baseline urinary symptoms, greater acute toxicity, larger prostate volume, higher $\mathrm{D}_{90}$, and age above 70 years were all associated with RTOG grade $\geq 2$ late urinary toxicity [23]. Late radiation proctitis has also been reported, with rates of rectal bleeding of 5-7\% and ulceration/fistula of $0.6 \%$ [24].

It is well-known that with increasing age, sexual function decreases [25]. Free text responses from the QoL questionnaires in our study suggest some men were less bothered about their sexual function with increasing age ("I'm over 75"), or due to their own or partner's co-morbidity. This may be an explanation that in time, patients appear to be less bothered about their decreased sexual function.

This study has limitations in that it was performed in a single-center, and baseline assessments of QoL were undertaken when neo-adjuvant had already been commenced in some patients. No interpretation of hormonal effects has therefore been undertaken. There was also some missing data. Although, data for the group mean HRQoL at the 10-year time point was known, the proportions of patients with small, moderate, and large declines in HRQoL at the 10-year time point was unfortunately not available. Our cohort size was relatively small in comparison to other studies and by 10 years, the response rate to the questionnaires had reduced to $48 \%$ meaning that the results at that timepoint may not be representative of the whole cohort. Nevertheless, this does represent a reasonable response rate, with a long time after the treatment. For comparison, a study by Sanda et al. had a response rate of $59 \%$ at only 2 years [5]. Similar to other publications, post-implant dosimetry was not correlated with urinary or bowel toxicity $[26,27]$.

\section{Conclusions}

Permanent prostate brachytherapy using ${ }^{125} \mathrm{I}$ has low toxicity up to 10 years after the treatment. When patients consider their treatment choice, they should be aware that their sexual function will temporarily deteriorate, and although improves, may not return to baseline levels in a long-term manner after the treatment. By 2 years, urinary toxicity appears to return to baseline level. Mild urinary and bowel symptoms are new at 10 years and may be either a late effect of brachytherapy or due to increasing age. QoL measurements should remain an integral part of clinical trials in the setting of localized prostate cancer.

\section{Disclosure}

The authors report no conflict of interest.

\section{References}

1. Cancer Research UK. Prostate cancer statistics. 06/05/2020. Available from: https://www.cancerresearchuk.org/healthprofessional/cancer-statistics/statistics-by-cancer-type/ prostate-cancer.

2. European Association of Urology. Prostate cancer 2019. 06/05/2020. Available from: https://uroweb.org/guideline/prostate-cancer/\#note_484.

3. Crook JM, Gomez-Iturriaga A, Wallace K et al. Comparison of health-related quality of life 5 years after SPIRIT: Surgical 
Prostatectomy Versus Interstitial Radiation Intervention Trial. J Clin Oncol 2011; 29: 362-368.

4. Donovan JL, Hamdy FC, Lane JA et al. Patient-reported outcomes after monitoring, surgery, or radiotherapy for prostate cancer. N Engl J Med 2016; 375: 1425-1437.

5. Hoffman KE, Penson DF, Zhao Z et al. Patient-reported outcomes through 5 years for active surveillance, surgery, brachytherapy, or external beam radiation with or without androgen deprivation therapy for localized prostate cancer. JAMA 2020; 323: 149-163.

6. Roeloffzen EM, Lips IM, van Gellekom MPR et al. Health-related quality of life up to six years after (125)I brachytherapy for early-stage prostate cancer. Int J Radiat Oncol Biol Phys 2010; 76: 1054-1060.

7. Henry AM, Al-Qaisieh B, Gould K et al. Outcomes following iodine-125 monotherapy for localized prostate cancer: the results of Leeds 10-year single-center brachytherapy experience. Int J Radiat Oncol Biol Phys 2010; 76: 50-56.

8. Wei JT, Dunn RL, Litwin MS et al. Development and validation of the expanded prostate cancer index composite (EPIC) for comprehensive assessment of health-related quality of life in men with prostate cancer. Urology 2000; 56: 899-905.

9. Pardo Y, Guedea F, Aguiló et al. Quality-of-life impact of primary treatments for localized prostate cancer in patients without hormonal treatment. J Clin Oncol 2010; 28: 4687-4696.

10. Sanda MG, Dunn RL, Michalski J et al. Quality of life and satisfaction with outcome among prostate-cancer survivors. N Engl J Med 2008; 358: 1250-1261.

11. Sylvester JE, Grimm PD, Eulau SM et al. Permanent prostate brachytherapy preplanned technique: The modern Seattle method step-by-step and dosimetric outcomes. Brachytherapy 2009; 8: 197-206.

12. Cohen J. Statistical power analysis for the behavioural sciences. $2^{\text {nd }}$ ed. Lawrence Erlbaum Associates, Hillsdale, NJ 1988.

13. Revicki D, Hays RD, Cella D et al. Recommended methods for determining responsiveness and minimally important differences for patient-reported outcomes. J Clin Epidemiol 2008; 61: 102-109.

14. Skolarus TA, Dunn RL, Sanda MG et al. Minimally important difference for the Expanded Prostate Cancer Index Composite Short Form. Urology 2015; 85: 101-105.

15. Ash D, Flynn A, Battermann J et al. ESTRO/EAU/EORTC recommendations on permanent seed implantation for localized prostate cancer. Radiother Oncol 2000; 57: 315-321.

16. Ash D, Bottomley D, Al-Qaisieh B et al. A prospective analysis of long-term quality of life after permanent I-125 brachytherapy for localised prostate cancer. Radiother Oncol 2007; 84: 135-139.

17. Lardas M, Liew M, van den Bergh RC et al. Quality of life outcomes after primary treatment for clinically localised prostate cancer: a systematic review. Eur Urol 2017; 72: 869-885.

18. Giberti C, Chiono L, Gallo F et al. Radical retropubic prostatectomy versus brachytherapy for low-risk prostatic cancer: a prospective study. World J Urol 2009; 27: 607-612.

19. Chen RC, Clark JA, Talcott JA. Individualizing quality-of-life outcomes reporting: how localized prostate cancer treatments affect patients with different levels of baseline urinary, bowel, and sexual function. J Clin Oncol 2009; 27: 3916-3922.

20. Roeloffzen EM, Battermann JJ, van Deursen MJH et al. Influence of dose on risk of acute urinary retention after iodine-125 prostate brachytherapy. Int J Radiat Oncol Biol Phys 2011; 80: 1072-1079.

21. Evers J, Kupper N, Kessing D et al. No long-term effects of hormonal therapy preceding brachytherapy on urinary function and health-related quality of life among prostate cancer patients. Urology 2010; 76: 1150-1156.
22. Grant JD, Litwin MS, Kwan L et al. Does hormone therapy exacerbate the adverse effects of radiotherapy in men with prostate cancer? A quality of life study. J Urol 2011; 185: 16741680.

23. Keyes M, Miller S, Pickles T et al. Late urinary side effects 10 years after low-dose-rate prostate brachytherapy: population-based results from a multiphysician practice treating with a standardized protocol and uniform dosimetric goals. Int J Radiat Oncol Biol Phys 2014; 90: 570-578.

24. Kishan AU, Kupelian PA. Late rectal toxicity after low-doserate brachytherapy: incidence, predictors, and management of side effects. Brachytherapy 2015; 14: 148-159.

25. Allan CA, Strauss BJ, McLachlan RI. Body composition, metabolic syndrome and testosterone in ageing men. Int J Impot Res 2007; 19: 448-457.

26. Neill M, Studer G, Le L et al. The nature and extent of urinary morbidity in relation to prostate brachytherapy urethral dosimetry. Brachytherapy 2007; 6: 173-179.

27. Williams SG, Millar JL, Duchesne GM et al. Factors predicting for urinary morbidity following 125iodine transperineal prostate brachytherapy. Radiother Oncol 2004; 73: 33-38. 